\title{
Some new derivatives of 5-aryl-, 2,5-diaryl- and 2-ethoxycarbonyl-5-aryl-indoles
}

\author{
Ioseb Sh. Chikvaidze, Nana N. Barbakadze, Shota A. Samsoniya \\ Division of Organic Chemistry, Ivane Javakhishvili Tbilisi State University, \\ 3 I. Chavchavadze Ave., Tbilisi, 0128, Georgia \\ E-mail: shota.samsonia@tsu.ge
}

\begin{abstract}
Some derivatives of 5-aryl-, 2.5-diaryl-, and 2-ethoxycarbonyl-5-aryl-indoles are synthesized. Some reactions of ethyl 5-phenylindole-2-carboxylate are described. It is revealed that an $\alpha$ ethoxycarbonyl group has a steric and electron-acceptor influence on indole substitution reactions. The structures of all novel compounds are confirmed by IR, UV, ${ }^{1} \mathrm{H} N \mathrm{NM},{ }^{13} \mathrm{C}$ NMR and elemental analyses.
\end{abstract}

Keywords: Indole, formylation, aminomethylation, nitrosation, N-alkylation

\section{Introduction}

Interest in indole and its derivatives arose at the beginning of the $20^{\text {th }}$ century, when it became known, that such compounds have a variety of physiological activities and play a vital role in the life processes of living organisms. ${ }^{1}$ The indole ring is present in many natural products and synthetic compounds having important medicinal properties. ${ }^{2 \mathrm{a}-\mathrm{c}}$ It is a structural element in many biologically and pharmaceutically active compounds with antibacterial, ${ }^{3 \mathrm{a}-\mathrm{c}}$ antimicrobial, ${ }^{4 \mathrm{a}, \mathrm{b}}$ antiinflammatory $^{5 \mathrm{a}, \mathrm{b}}$ and other activities. ${ }^{6 \mathrm{a}-\mathrm{c}}$ Indolic compounds are also known as antidepressants, tranquilizers and analgetics. $^{7}$

Earlier, 5-arylindole derivatives were obtained as by-products of bicyclization of 4,4'diarylendihydrazones with yield in $3-10 \% .{ }^{8 \mathrm{a}-\mathrm{d}}$ Such low yields complicated further study of these substances. We synthesized 5-aryl-indoles and 2-aryl-indoles by the Fischer reaction using the corresponding arylhydrazones $^{9 \mathrm{a}-\mathrm{g}}$ and studied their properties. Some 2-aryl-indoles show high pharmacological activity. ${ }^{4 \mathrm{~b}, 10,11}$ The activity against hormone-dependent carcinoma and fluorescent and intercalation properties of the mentioned substances are of especial interest. ${ }^{12 \mathrm{a}-\mathrm{e}}$

Among 5-phenyl-indoles, some highly active substances were discovered showing the ability to inhibit serotonin receptors $5-\mathrm{HT}_{1 \mathrm{~A}}$ and $5-\mathrm{HT}_{2}{ }^{13}$ and plasminogen activator inhibitors. ${ }^{14}$ The 
latter is of great importance for treatment of acute arterial thrombosis, atherosclerosis, diabetes, cardiovascular disease, etc.

\section{Results and Discussion}

In continuation of our research our interest was to synthesize a series of new derivatives containing one or two phenyl groups.

Our study showed that the Fischer synthesis ${ }^{15 a-c}$ is the most efficient method for the preparation of 5-aryl- and 2-ethoxycarbonyl-5-aryl-indoles. We have tested almost every reported method ${ }^{16}$ for the synthesis of indole derivatives, but the best results were achieved using polyphosphoric acid (PPA) as a catalyst in the Fischer reaction. In comparison to the published methods, Fischer's method reduces the number of steps for indole formation allowing us to carry out one-step reactions without isolation of intermediates, often unstable hydrazones. 2,5-Diarylindoles 3a-d were prepared in one step by reaction of arylhydrazine $\mathbf{1}$ with a corresponding ketone 2a-d in PPA (Scheme 1, Table 1). Various other condensing agents frequently applied in Fischer's method, such as dry $\mathrm{HCl} /$ ethanol, $\mathrm{H}_{2} \mathrm{SO}_{4}$ /ethanol, $\mathrm{H}_{2} \mathrm{SO}_{4}$ /acetic acid, etc., were inefficient in these reactions. The yields of products 3a-d were in the range 12-40\%.

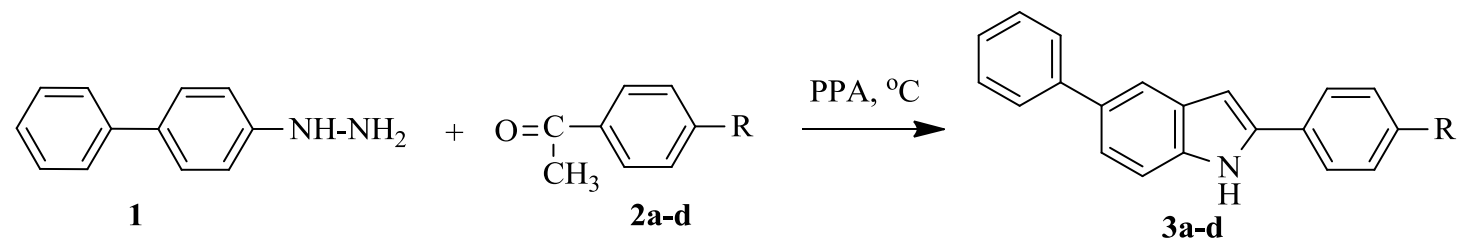

Scheme 1. Synthesis of the indole systems 3a-d.

Table 1. Conditions and yields of 2,5-diaryl-indoles 3a-d

\begin{tabular}{cccc}
\hline Reactant & $\mathrm{R}$ & temp ${ }^{\circ} \mathrm{C}$ & $\begin{array}{l}\text { Product, } \\
\text { Yield }(\%)\end{array}$ \\
\hline $\mathbf{2 a}$ & $\mathrm{H}$ & 80 & $\mathbf{3 a}, 27$ \\
$\mathbf{2 b}$ & $\mathrm{OCH}_{3}$ & 85 & $\mathbf{3 b}, 30$ \\
$\mathbf{2 c}$ & $\mathrm{NO}_{2}$ & 80 & $\mathbf{3 c}, 12$ \\
$\mathbf{2 d}$ & $\left(\mathrm{CH}_{2}\right)_{2} \mathrm{C}_{6} \mathrm{H}_{5}$ & 110 & $\mathbf{3 d}, 30$ \\
\hline
\end{tabular}

The reaction of 4-hydrazinobiphenyl and 4-acetylbiphenyl in polyphosphoric acid did not form the expected indole system. The reaction was complicated due to side processes. Biphenyl sublimed from the reaction mixture, most probably produced by decomposition of an unstable intermediate hydrazone. 
We were also unable to obtain ethyl 5-phenylindole-2-carboxylate 6 under the same conditions. This compound was not firmed in polyphosphoric acid neither with the above described method nor by the preliminary isolation of hydrazone. The best result was achieved when the reaction was carried out using ethyl esters of polyphosphoric acid (PPAEE). The reaction was carried out in two steps. We first isolated the biphenylhydrazone 5 of ethyl pyruvate, which by cyclization at $80{ }^{\circ} \mathrm{C}$ gave chromatographically pure compound 6 in $58 \%$ yield (Scheme 2).
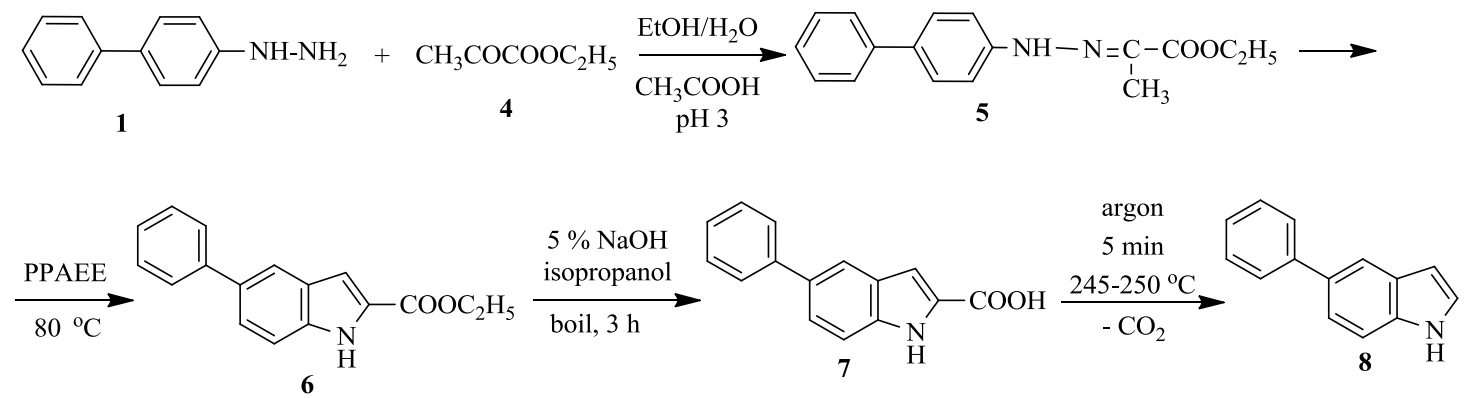

Scheme 2. Synthesis of ethyl 5-phenylindole-2-carboxylate 6 and conversion of ester group.

From the synthesized compounds we selected ethyl 5-phenylindole-2-carboxylate and studied its chemical properties in two ways: (1) manipulation of the ester group (Scheme 2) and (2) electrophilic substitution reactions (Scheme 3) in order to define the nature of the influence of the substituents on the reaction processes.

Saponification of the ester group and thermal decarboxylation of acid 7 was described earlier. ${ }^{17}$ The use of an inert gas by us increased the yield of compound $\mathbf{8}$ to $55 \%$ (Scheme 2).

For ethyl 5-phenylindole-2-carboxylate we carried out some classical electrophilic substitution reactions of the indole ring, such as formylation according to Vilsmeier-Haack, ${ }^{18}$ dimethylaminomethylation according to Mannich, ${ }^{19}$ nitrosation, ${ }^{18,20}$ azo-coupling ${ }^{21 \mathrm{a}, \mathrm{b}}$ and $\mathrm{N}$ alkylation. $^{22 a, b}$ 


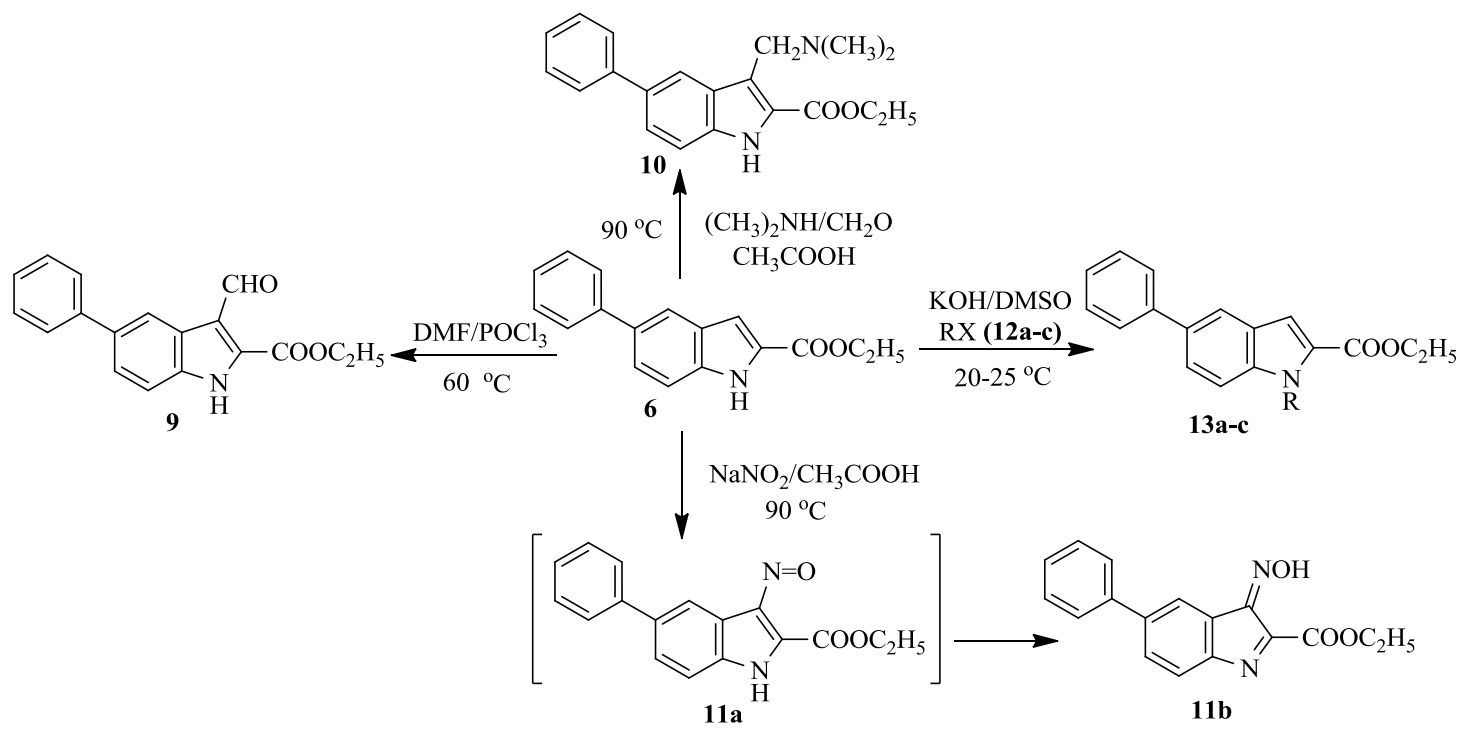

Scheme 3. Electrophilic substitution reactions of ethyl 5-phenylindole-2-carboxylate 6

Table 2. Conditions and yields of N-alkylindoles 13a-c

\begin{tabular}{|c|c|c|c|c|c|}
\hline \multicolumn{2}{|c|}{$\mathrm{RX}$} & \multicolumn{2}{|c|}{$\begin{array}{c}\text { Product } \\
\mathrm{R} \\
\end{array}$} & \multirow{2}{*}{$\frac{\operatorname{Temp}^{\circ} \mathrm{C}}{20}$} & \multirow{2}{*}{$\begin{array}{c}\text { Yield (\%) } \\
90\end{array}$} \\
\hline $\mathrm{CH}_{3} \mathrm{I}$ & $12 a$ & $\mathrm{CH}_{3}$ & 13a & & \\
\hline $\mathrm{C}_{2} \mathrm{H}_{5} \mathrm{CI}$ & $12 b$ & $\mathrm{C}_{2} \mathrm{H}_{5}$ & 13b & 20 & 92 \\
\hline $\mathrm{C}_{4} \mathrm{H}_{9} \mathrm{~B}_{\mathrm{r}}$ & $12 \mathrm{c}$ & $\mathrm{C}_{4} \mathrm{H}_{9}$ & $13 \mathrm{c}$ & 20 & 87 \\
\hline
\end{tabular}

Ethyl 5-phenylindole-2-carboxylate does not enter in the azo-coupling reaction - we suppose that this failure is caused by the acceptor influence of the ester group. It also affects the nitrosation reaction, Vilsmeier-Haack formylation and Mannich aminomethylation (Scheme 3) which proceed only at temperatures 90,60 and $90{ }^{\circ} \mathrm{C}$ respectively. Considering the low reactivity of ethyl 5-phenylindole-2-carboxylate $\mathbf{6}$ at low temperatures, formylation was carried out without previous preparation of formylation complex, which noticeably simplifies the process. Formylation of compound 6 proceeded at $60{ }^{\circ} \mathrm{C}$ for 3 hours and gave 3 -formyl derivative 9 with a yield of $88 \%$.

Dimethylaminomethylation of ethyl 5-phenylindole-2-carboxylate 6 with a freshly prepared mixture of dimethylamine, formalin and acetic acid also proceeded at high temperature, at $90^{\circ} \mathrm{C}$, and resulted in gramine-type Mannich base 10 with the yield of 65\%.

The ester group has no effect on $\mathrm{N}$-alkylation (Scheme 3), which we conducted in a superbasic medium similar to that described ${ }^{22 a, b}$ and obtained the corresponding $\mathrm{N}$-alkyl derivatives 13a-c (Scheme 3, Table 2). The ester group does appear to affect the isomerization of 
the nitrosation product, which was obtained in the form of an isonitroso derivative, 11, in $90 \%$ yield. Apparently isonitroso form is favoured, perhaps by an intramolecular hydrogen bond between the oxime hydrogen and the neighboring ethoxycarbonyl group. A signal (singlet, 13.05 $\mathrm{ppm}$ ) in the ${ }^{1} \mathrm{H}$ NMR spectrum of the nitrosation product could be characteristic of an oxime 11b hydrogen atom, but it cold also be characteristic of an indole $\mathrm{N}-\mathrm{H}$ proton of nitroso derivative 11a (analogous to a singlet signal at $12.88 \mathrm{ppm}$ in the ${ }^{1} \mathrm{H}$ NMR spectrum of formyl derivative 9). Similar isomerization characterizes other N-non-substituted 3-nitrosoindoles. ${ }^{18,20 \mathrm{~b}-\mathrm{e}}$ Structure 11b is confirmed also by the fact that this compound has no UV absorption maxima in longwavelength range whereas the $\mathrm{N}$-methyl-3-nitroso-2-phenylindoles, synthesized by us earlier, have maxima in 368-370 $\mathrm{nm}$ range. ${ }^{20 \mathrm{f}}$ Such compounds form of green crystals. ${ }^{20 \mathrm{f}}$

By heating a mixture of aldehyde 9 and hydrazine hydrate in glacial acetic acid, 8-phenyl3,5-dihydro-4H-pyridazino[4,5-b]indol-4-one 15 was formed in 92\% yield (Scheme 4).

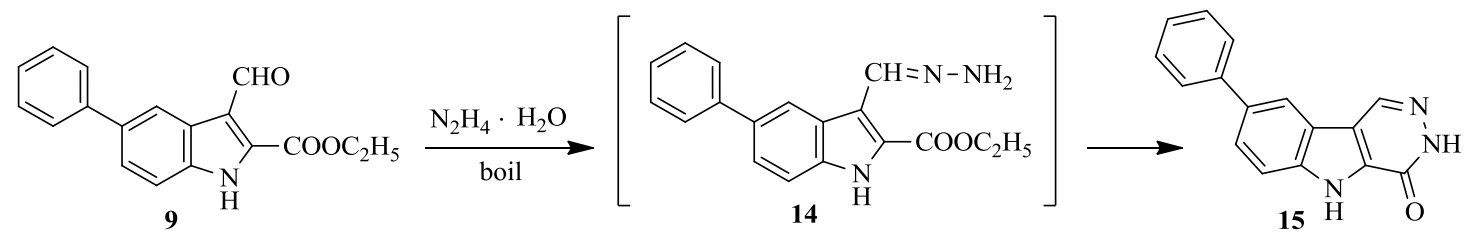

Scheme 4. Synthesis of 8-phenyl-3,5-dihydro-4H-pyridazino[4,5-b]indol-4-one 15.

\section{Conclusions}

In order to search for compounds with useful properties, new indole-containing heterocyclic compounds were synthesized, in particular, 5-aryl- and 2,5-diaryl-indoles and 2-ethoxycarbonyl5-aryl-indoles. The preparative method for their synthesis utilized an arylhydrazine and a carbonyl compound in polyphosphoric acid without isolation of intermediate hydrazone.

Taking into consideration the results obtained it can be concluded that the 5-phenyl group has no effect on the reactions of these indoles. The reactivity is however significantly reduced by the electronic and steric influence of an $\alpha$-ethoxycarbonyl group, and electrophilic substitution reactions proceeded only at high temperatures. $\beta$-Substituted products were obtained in all cases.

\section{Experimental Section}

General. Conversion of reactants and purity of the obtained compounds were monitored by TLC on silufol UV-254 plates. Melting points were determined on a NAGEMA PHMK 05 apparatus. The IR spectra were recorded on a Thermo Nicolet FTIR photometer "AVATAR 370". The UV spectra were obtained on an UV/Vis Varian "CARRY-100" instrument. Elemental analyses were carried out on a Multi EA 2000 analyzer. ${ }^{1} \mathrm{H}$ NMR and ${ }^{13} \mathrm{C}$ NMR were recorded on a BRUKER 
DRX-500 (500 MHz) and VARIAN MERCURY $300 \mathrm{VX}(300 \mathrm{MHz})$ in DMSO- $d_{6}$ (with TMS for ${ }^{1} \mathrm{H}$ and ${ }^{13} \mathrm{C}$ as the internal standard).

\section{General procedure for preparation derivatives of 2,5-diarylindoles (3a-d)}

A mixture of acetophenone derivatives 2a-d $(10 \mathrm{mmol})$, 4-hydrazinobiphenyl hydrochloride 1 (2.65 g, $12 \mathrm{mmol}$ ) and PPA (40 g) was stirred at the specified temperature (Table 1) for 45 minutes. After the reaction was complete, the reaction mixture was cooled to room temperature and poured onto crushed ice (200 g) with stirring. The precipitate obtained was filtered off and washed with water until neutral and dried. The crude product was purified by column chromatography (silica gel) $\mathbf{3 b}, \mathbf{3 c}$ or recrystallized 3a, 3d to gives the corresponding 2,5diarylindoles 3a-d.

2,5-Diphenylindole (3a). Yield 27\%; colorless crystals (from benzene/hexane, 1:3); mp $196{ }^{\circ} \mathrm{C}$ (lit. ${ }^{23} \mathrm{mp} \mathrm{192-193}{ }^{\circ} \mathrm{C}$ ); UV $\lambda_{\max } / \mathrm{nm}$ (Ethanol), (log $\varepsilon$ ) 215 (4.4), 223 (4.58), 272 (4.2), 323 (3.52). IR (Vaseline oil) $v_{\max } / \mathrm{cm}^{-1}: 3410(\mathrm{NH}) ;{ }^{1} \mathrm{H}$ NMR (DMSO- $\left.d_{6}, 300 \mathrm{MHz}\right) \delta 7.22-7.47$ (m, $6 \mathrm{H}), 7.23(\mathrm{~d}, J=8.9 \mathrm{~Hz}, 1 \mathrm{H}), 7.34(\mathrm{dd}, J=1.8 \mathrm{~Hz}, J=8.9 \mathrm{~Hz}, 1 \mathrm{H}), 7.60(\mathrm{~d}, J=8.2 \mathrm{~Hz}, 2 \mathrm{H})$, $7.71(\mathrm{~d}, J=1.8 \mathrm{~Hz}, 1 \mathrm{H}), 7.8(\mathrm{~d}, J=2.2 \mathrm{~Hz}, 1 \mathrm{H}), 7.82(\mathrm{~d}, J=8.5 \mathrm{~Hz}, 2 \mathrm{H}), 11.3(\mathrm{~s}, 1 \mathrm{H})$. Anal. Calcd for $\mathrm{C}_{20} \mathrm{H}_{15} \mathrm{~N}$ : C, 89.19; H, 5.61; N, 5.20. Found: C, 89.41; H, 5.66; N, 5.12.

2-(p-Methoxyphenyl)-5-phenylindole (3b). Yield 30\%; colorless crystals (from benzene); mp 210-211 ${ }^{\circ} \mathrm{C} ; \mathrm{R}_{\mathrm{f}} 0.9$ benzene; UV $\lambda_{\max } / \mathrm{nm}$ (Ethanol), (loge) 214 (4.54), 227 (4.46), 286 (3.48), 316 (3.34), IR (Vaseline oil) $v_{\max } / \mathrm{cm}^{-1}: 3425(\mathrm{NH}) ;{ }^{1} \mathrm{H}$ NMR (DMSO- $\left.d_{6}, 300 \mathrm{MHz}\right) \delta 3.82(\mathrm{~s}$, $3 \mathrm{H}), 7.06(\mathrm{~d}, J=1.8 \mathrm{~Hz}, 1 \mathrm{H}), 7.21(\mathrm{t}, 7.1 \mathrm{~Hz}, 1 \mathrm{H}), 7.25$ (d, $J=7.1 \mathrm{~Hz}, 2 \mathrm{H}), 7.3-7.39(\mathrm{~m}, 4 \mathrm{H})$, $7.40(\mathrm{dd}, J=1.6, J=8.8 \mathrm{~Hz}, 1 \mathrm{H}), 7.48(\mathrm{~d}, J=8.8 \mathrm{~Hz}, 1 \mathrm{H}), 7.75(\mathrm{~d}, J=1.6 \mathrm{~Hz}, 1 \mathrm{H}), 8.06(\mathrm{~d}, J$ $=7.1 \mathrm{~Hz}, 2 \mathrm{H}), 11.3(\mathrm{~s}, 1 \mathrm{H})$. Anal. Calcd for $\mathrm{C}_{21} \mathrm{H}_{17} \mathrm{NO}: \mathrm{C}, 84.25 ; \mathrm{H}, 5.72 ; \mathrm{N}, 4.68$. Found: $\mathrm{C}$, 84.34; H, 5.91; N, 4.43.

2-(p-Nitrophenyl)-5-phenylindole (3c). Yield 12\%; yellow crystals (from benzene), mp $240{ }^{\circ} \mathrm{C}$; $\mathrm{R}_{\mathrm{f}} 0.36$ benzene; UV $\lambda_{\max } / \mathrm{nm}$ (Ethanol), (loge) 212 (4.44), 229 (4.45), 280 (3.40), 316 (3.34). IR (Vaseline oil) $v_{\max } / \mathrm{cm}^{-1}: 3430(\mathrm{NH}), 1350\left(\mathrm{NO}_{2}\right)$; ${ }^{1} \mathrm{H}$ NMR (DMSO-d, $\left.300 \mathrm{MHz}\right) \delta 7.07(\mathrm{~d}, J=$ $1.8 \mathrm{~Hz}, 1 \mathrm{H}), 7.23$ (t, $J=7.1 \mathrm{~Hz}, 1 \mathrm{H}), 7.32$ (t, $J=7.1 \mathrm{~Hz}, 2 \mathrm{H}), 7.38$ (dd, $J=1.6 \mathrm{~Hz}, J=8.8 \mathrm{~Hz}, 1 \mathrm{H})$, $7.48(\mathrm{~d}, J=8.8 \mathrm{~Hz}, 1 \mathrm{H}), 7.60(\mathrm{~d}, J=7.1 \mathrm{~Hz}, 2 \mathrm{H}), 7.76(\mathrm{~d}, J=1.6 \mathrm{~Hz}, 1 \mathrm{H}), 8.06(\mathrm{~d}, J=7.1 \mathrm{~Hz}$, $2 \mathrm{H}), 8.27(\mathrm{~d}, J=7.1 \mathrm{~Hz}, 2 \mathrm{H}), 11.6(\mathrm{~s}, 1 \mathrm{H}) ;{ }^{13} \mathrm{C}$ NMR $\left(\mathrm{DMSO}_{6}, 75 \mathrm{MHz}\right) \delta 102.1,111.5$, $118.5,122.2$, 123.6, 125.0, 125.6, 126.5, 128.0, 128.7, 132.5, 135.6, 137.3, 138.4, 141.7, 145.6. Anal. Calcd for $\mathrm{C}_{20} \mathrm{H}_{14} \mathrm{~N}_{2} \mathrm{O}_{2}$ : C, 76.42; H, 4.49; N, 8.91. Found: C, 76.65; H, 4.44; N, 8.78.

5-Phenyl-2-[4-(2-phenylethyl)phenyl]indole (3d). Yield 30\%; colorless crystals (from benzene), mp 200-201 ${ }^{\circ} \mathrm{C} ; \mathrm{R}_{\mathrm{f}} 0.32$ hexane: diethyl ether $=2: 1(\mathrm{v} / \mathrm{v}) ; \mathrm{UV} \lambda_{\max } / \mathrm{nm}$ (Ethanol), (loge) 215 (4.41), 245 (4.13), 313 (4.30). IR (Vaseline oil) $v_{\max } / \mathrm{cm}^{-1}: 3452$ (NH); ${ }^{1} \mathrm{H}$ NMR $\left(\mathrm{DMSO}-d_{6}, 300 \mathrm{MHz}\right) \delta 2.94(\mathrm{~s}, 4 \mathrm{H}), 6.74(\mathrm{~d}, J=2.4 \mathrm{~Hz}, 1 \mathrm{H}), 7.29$ (dd, $J=2.0 \mathrm{~Hz}, J=8.3 \mathrm{~Hz}$, $1 \mathrm{H}), 7.37$ (t, $J=7.2 \mathrm{~Hz}, 2 \mathrm{H}), 7.42(\mathrm{~d}, J=8.3 \mathrm{~Hz}, 1 \mathrm{H}), 7.54(\mathrm{t}, J=7.2 \mathrm{~Hz}, 1 \mathrm{H}), 7.58$ (dd, $J=2.0$ $\mathrm{Hz}, J=7.2 \mathrm{~Hz}, 2 \mathrm{H}), 7.68(\mathrm{~d}, J=2.0 \mathrm{~Hz}, 1 \mathrm{H}), 7.72(\mathrm{~d}, J=8.8 \mathrm{~Hz}, 2 \mathrm{H}), 7.80(\mathrm{~d}, J=8.8 \mathrm{~Hz}, 2 \mathrm{H})$, 11.23 (s, 1H). Anal. Calcd for $\mathrm{C}_{28} \mathrm{H}_{23} \mathrm{~N}$ : C, 90.04; H, 6.21; N, 3.75. Found: C, 90.31; H, 6.17; N, 3.97 . 
Biphenylhydrazone of ethyl pyruvate (5). To the mixture of ethyl pyruvate 4 (5.8 g, $50 \mathrm{mmol})$ in ethanol $(40 \mathrm{ml})$ a solution of the hydrochloride of 4-hydrazinobiphenyl 1 (11.9 g, $54 \mathrm{mmol})$ was added at $40-50{ }^{\circ} \mathrm{C}$ in water $(500 \mathrm{ml})$, then acetic acid (4-5 drops) was added dropwise $(\mathrm{pH}$ 3 ). The reaction mixture was mixed for $2 \mathrm{~h}$ at room temperature. The precipitated solid was filtered off, washed with water until neutral and dried. The yellow crystals were dissolved in benzene and precipitated by addition of small amount of hexane, then filtered, washed with hexane and dried again. Yield 57\%; yellowish crystals; mp 97-98 ${ }^{\circ} \mathrm{C} ; \mathrm{R}_{\mathrm{f}} 0.15$ benzene; UV $\lambda_{\max } / \mathrm{nm}$, (Ethanol), (loge) 204 (4.7), 267 (4.3), 338 (4.9); IR (KBr) $v_{\max } / \mathrm{cm}^{-1}$ : 3290-3550 (NH), $1674(\mathrm{C}=\mathrm{O}), 1620(\mathrm{C}=\mathrm{N}) ;{ }^{1} \mathrm{H}$ NMR $\left(\mathrm{DMSO}-d_{6}, 500 \mathrm{MHz}\right) \delta 1.27(\mathrm{t}, J=7.1 \mathrm{~Hz}, 3 \mathrm{H}), 2.08(\mathrm{~s}$, $3 \mathrm{H}), 4.19$ (q, $J=7.1 \mathrm{~Hz}, 2 \mathrm{H}), 7.28-7.65(\mathrm{~m}, 6 \mathrm{H}), 7.29(\mathrm{t}, J=7.4 \mathrm{~Hz}, 1 \mathrm{H}), 7.42$ (t, $J=7.4 \mathrm{~Hz}$, 1H), $9.94(\mathrm{~s}, 1 \mathrm{H}) ;{ }^{13} \mathrm{C}$ NMR (DMSO-D, $\left.125 \mathrm{MHz}\right) \delta 11.8,14.3,60.2,114.1,125.9,126.6$, 127.3, 128.8, 128.9, 132.1, 132.6, 139.9, 143.9, 164.9. Anal. Calcd for $\mathrm{C}_{17} \mathrm{H}_{18} \mathrm{~N}_{2} \mathrm{O}_{2}$ : C, 72.32; $\mathrm{H}$, $6.43 ;$ N, 9.92. Found: C, 72.13; H, 6.61; N, 9.71.

Ethyl 5-phenylindole-2-carboxylate (6). The mixture of biphenylhydrazone of ethyl pyruvate 5 (5.64 g, $20 \mathrm{mmol}$ ) and PPAEE (60 g) was heated at $80{ }^{\circ} \mathrm{C}$ during $20 \mathrm{~min}$. The reaction mixture was cooled, poured into ice water $(600 \mathrm{ml})$, and filtered. The solid was washed with water until neutral and dried. The purification was carried out by column chromatography (silica gel), eluent - hexane. Yield 58\%; colorless crystals, mp $174-175{ }^{\circ} \mathrm{C}$ (lit. ${ }^{17} \mathrm{mp} 174-175{ }^{\circ} \mathrm{C}$ ); $\mathrm{R}_{\mathrm{f}} 0.8$ acetone: benzene $=1: 5(\mathrm{v} / \mathrm{v}) ;$ UV $\lambda_{\max } / \mathrm{nm}$ (Benzene), (loge) 206 (4.3), 260 (4.5), 300 (4.1); IR (KBr) $\nu_{\max } / \mathrm{cm}^{-1}: 3310(\mathrm{NH}), 1690(\mathrm{C}=\mathrm{O}) ;{ }^{1} \mathrm{H}$ NMR (DMSO- $\left.d_{6}, 500 \mathrm{MHz}\right) \delta 1.30(\mathrm{t}, J=7.3 \mathrm{~Hz}, 3 \mathrm{H})$, 4.32 (q, $J=7.3 \mathrm{~Hz}, 2 \mathrm{H}), 7.12(\mathrm{~d}, J=0.8 \mathrm{~Hz}, 1 \mathrm{H}), 7.3-7.6(\mathrm{~m}, 7 \mathrm{H}), 7.81(\mathrm{~s}, 1 \mathrm{H}), 11.85(\mathrm{~s}, 1 \mathrm{H})$. Anal. Calcd. for $\mathrm{C}_{17} \mathrm{H}_{15} \mathrm{NO}_{2}$ : C, 76.98; H, 5.66; N, 5.28. Found: C, 76.95; H, 5.65; N, 5.35.

5-Phenylindole-2-carboxylic acid (7). A suspension of ethyl 5-phenylindole-2-carboxylate 6 $(2.65 \mathrm{~g}, 10 \mathrm{mmol}), 5 \%$ solution of sodium hydroxide $(700 \mathrm{ml})$ and isopropanol (100 ml) was boiled for $3 \mathrm{~h}$. The solution is cooled, filtered and acidified with $\mathrm{HCl}(\mathrm{pH} 1)$. The solid was filtered off, washed with water until neutral and dried. Yield 80\%; mp $246{ }^{\circ} \mathrm{C}$ (lit. ${ }^{17} \mathrm{mp} 246{ }^{\circ} \mathrm{C}$ ); $\mathrm{R}_{\mathrm{f}} 0.61$ benzene $:$ diethyl ether $=2: 1,(\mathrm{v} / \mathrm{v}) ; \mathrm{UV} \lambda_{\max } / \mathrm{nm}$ (Ethanol), (loge) 206 (4.40), 261 (4.61), 300 (4.06); IR (Vaseline oil) $v_{\max } / \mathrm{cm}^{-1}: 3380,3330(\mathrm{NH}), 1715,1675$ (C= O). ${ }^{1} \mathrm{H}$ NMR $\left(\mathrm{DMSO}-d_{6}, 300 \mathrm{MHz}\right) \delta 7.06(\mathrm{~d}, J=1.6 \mathrm{~Hz}, 1 \mathrm{H}), 7.2-7.6(\mathrm{~m}, 7 \mathrm{H}), 7.78(\mathrm{~d}, J=1.6 \mathrm{~Hz}, 1 \mathrm{H})$, $11.55(\mathrm{~s}, 1 \mathrm{H}) ;{ }^{13} \mathrm{C}$ NMR (DMSO-D $\left.6,75 \mathrm{MHz}\right) \delta 106.8,112.5,119.4,123.0,125.6,126.5,127.3$, 128.0, 130.1, 132.3, 136.4, 141.7, 163.5. Anal. Calcd for $\mathrm{C}_{15} \mathrm{H}_{11} \mathrm{NO}_{2}$ : N 5.91. Found: N, 5.90.

5-Phenylindole (8). 5-phenylindole-2-carboxylic acid 7 (1 g, $4.2 \mathrm{mmol})$ was heated in a stream of argon for 5 minutes at $245-250{ }^{\circ} \mathrm{C}$. The product was purified by column chromatography (silica gel) eluting with hexane. Yield 55\%; mp 70-71 ${ }^{\circ} \mathrm{C}$ (lit. ${ }^{17} \mathrm{mp} 70-71{ }^{\circ} \mathrm{C}$ ). $\mathrm{R}_{\mathrm{f}} 0.63$ benzene; UV $\lambda_{\max } / \mathrm{nm}$ (ethanol), (loge) 207 (4.39), 252 (4.56), 283 (3.97); IR (vaseline oil) $v_{\max } / \mathrm{cm}^{-1}: 3490$ (NH); Anal. Calcd. for $\mathrm{C}_{14} \mathrm{H}_{11} \mathrm{~N}$ : C, 87.1; H, 5.7. Found: C, 87.4; H, 5.8.

Ethyl 3-formyl-5-phenylindole-2-carboxylate (9). To a solution of ethyl 5-phenylindole-2carboxylate $6(4.24 \mathrm{~g}, 16 \mathrm{mmol})$ cooled to $2-5^{\circ} \mathrm{C}$ in freshly distilled dimethylformamide (DMF) $(40 \mathrm{ml}), \mathrm{POCl}_{3}(12.3 \mathrm{~g}, 80 \mathrm{mmol})$ was added dropwise. The formed suspension was stirred during $3 \mathrm{~h}$ at $60{ }^{\circ} \mathrm{C}$. Then it was then cooled and added to crushed ice $(200 \mathrm{~g})$, then a $25 \%$ 
solution of $\mathrm{NaOH}$ ( $\mathrm{pH} 10$ ) was added. The precipitate obtained was filtered off, washed with water until neutral, dried and recrystallised from benzene. Yield 88\%; light yellow crystals; mp > $220{ }^{\circ} \mathrm{C}$ (decomp.); $\mathrm{R}_{\mathrm{f}} 0.53$ benzene : diethyl ether $=2: 1$, (v/v); UV $\lambda_{\max } / \mathrm{nm}$ (Ethanol), (loge) 214 (3.6), 268 (4.3), 329 (4.1); IR (KBr) $v_{\max } / \mathrm{cm}^{-1}: 3178(\mathrm{NH}), 1636(\mathrm{C}=\mathrm{O}) .{ }^{1} \mathrm{H}$ NMR (DMSO- $d_{6}$, $500 \mathrm{MHz}) \delta 1.40(\mathrm{t}, J=7.6 \mathrm{~Hz}, 3 \mathrm{H}), 4.46(\mathrm{q}, J=7.6 \mathrm{~Hz}, 2 \mathrm{H}), 7.36(\mathrm{t}, J=7.0 \mathrm{~Hz}, 1 \mathrm{H}), 7.48(\mathrm{t}, J$ $=7.0 \mathrm{~Hz}, 2 \mathrm{H}), 7.64(\mathrm{~d}, J=8.5 \mathrm{~Hz}, 1 \mathrm{H}), 7.65(\mathrm{~d}, J=8.5 \mathrm{~Hz}, 1 \mathrm{H}), 8.47(\mathrm{~s}, 1 \mathrm{H}), 10.67(\mathrm{~s}, 1 \mathrm{H})$, 12.88 (s, 1H). ${ }^{13} \mathrm{C}$ (DMSO-D, $125 \mathrm{MHz}$ ) NMR $\delta$ 14.3, 62.1, 113.9, 118.8, 120.3, 125.6, 125.7, 127.1, 127.3, 129.2, 133.5, 135.5, 136.3, 140.9, 160.3, 187.8. Anal. Calcd for $\mathrm{C}_{18} \mathrm{H}_{15} \mathrm{NO}_{3}$ : C, 73.71; H, 5.15; N, 4.78. Found: C, 73.44; H, 5.34; N, 4.98.

Ethyl 3-dimethylaminomethyl-5-phenylindole-2-carboxylate (10). To the solution of (4 g, 15 mmol) ethyl 5-phenylindole-2-carboxylate 6 in glacial acetic acid $(15 \mathrm{~mL})$ a solution prepared from $33 \%$ aqueous dimethylamine $(11 \mathrm{ml}, 80 \mathrm{mmol})$, glacial acetic acid $(15 \mathrm{~mL})$ and formalin $(6$ $\mathrm{mL}, 75 \mathrm{mmol}, 40 \%$ ) were added. The mixture was stirred for $2 \mathrm{~h}$ at $90{ }^{\circ} \mathrm{C}$, cooled and poured onto crushed ice $(200 \mathrm{~g})$, then $25 \% \mathrm{NaOH}$ was added o bring the $\mathrm{pH}$ to 10 . The precipitate was filtered off, washed with water until $\mathrm{pH} \mathrm{7,} \mathrm{dried} \mathrm{over} \mathrm{KOH}$ and recrystallized from benzene. Yield 62\%; colorless crystals; mp $128-129^{\circ} \mathrm{C} ; \mathrm{R}_{\mathrm{f}} 0.30$ benzene; $\mathrm{UV} \lambda_{\max } / \mathrm{nm}$ (ethanol), $(\log \varepsilon)$ 206 (4.2), 261 (4.4), 306 (3.9); IR (KBr) $v_{\max } / \mathrm{cm}^{-1}$ : 3317 (NH), 2800-3000 (CH), 1674 (C=O); ${ }^{1} \mathrm{H}$ NMR (DMSO-d, $\left.500 \mathrm{MHz}\right) \delta 1.36(\mathrm{t}, J=6.9 \mathrm{~Hz}, 3 \mathrm{H}), 2.18(\mathrm{~s}, 3 \mathrm{H}), 3.95(\mathrm{~s}, 3 \mathrm{H}), 4.34(\mathrm{q}, J=$ $6.9 \mathrm{~Hz}, 2 \mathrm{H}), 7.32(\mathrm{t}, J=6.5 \mathrm{~Hz}, 1 \mathrm{H}), 7.46(\mathrm{t}, J=6.5 \mathrm{~Hz}, 2 \mathrm{H}), 7.51(\mathrm{~d}, J=8.5 \mathrm{~Hz}, 1 \mathrm{H}), 7.57(\mathrm{~d}, J$ $=8.5 \mathrm{~Hz}, 1 \mathrm{H}), 7.65(\mathrm{~d}, J=6.5 \mathrm{~Hz}, 2 \mathrm{H}), 8.02(\mathrm{~s}, 1 \mathrm{H}), 11.68(\mathrm{~s}, 1 \mathrm{H}) .{ }^{13} \mathrm{C}\left(\mathrm{DMSO}_{6}, 125 \mathrm{MHz}\right)$ NMR $\delta$ 14.3, 45.1, 52.4, 60.3, 112.8, 119.3, 124.3, 125.1, 126.5, 126.7, 128.8, 132.1, 135.7, 141.4, 161.7. Anal. Calcd for $\mathrm{C}_{20} \mathrm{H}_{22} \mathrm{~N}_{2} \mathrm{O}_{2}$ : C, 74.51; H, 6.88; N, 8.69. Found: C, 74.84; H, 6.46; N, 8.91.

Ethyl 3-(hydroxyimino)-5-phenyl-3H-indole-2-carboxylate (11b). To a solution (0.26 g, 10 mmol) of ethyl 5-phenylindole-2-carboxylate 6 in acetic acid (200 ml) $\mathrm{NaNO}_{2}(1 \mathrm{~g}, 15 \mathrm{mmol})$ was added and the mixture stirred for $2 \mathrm{~h}$ at $90{ }^{\circ} \mathrm{C}$. The solution was cooled, cold water (1 l) was added and the resulting mixture saturated with anhydrous $\mathrm{Na}_{2} \mathrm{SO}_{4}$. The precipitate was filtered off, washed with water until $\mathrm{pH} 7$ and dried. Yield 90\%; yellowish crystals; mp 159-160 ${ }^{\circ} \mathrm{C} ; \mathrm{R}_{\mathrm{f}}$ 0.30 benzene; UV $\lambda_{\max } / \mathrm{nm}$ (DMF), (loge) 209 (4.5), 223 (3.95), 252 (4.53); IR (Vaseline oil) $v_{\max } / \mathrm{cm}^{-1}: 3300-3600(\mathrm{OH}) ;{ }^{1} \mathrm{H}$ NMR (DMSO- $\left.d_{6}, 300 \mathrm{MHz}\right) \delta 1.45(\mathrm{t}, J=6.6 \mathrm{~Hz}, 3 \mathrm{H}), 4.45(\mathrm{q}$, $2 \mathrm{H}, J=6.6 \mathrm{~Hz}), 7.33(\mathrm{~d}, J=8.5 \mathrm{~Hz}, 1 \mathrm{H}), 7.42(\mathrm{~d}, J=8.5 \mathrm{~Hz}, 1 \mathrm{H}), 7.44(\mathrm{t}, J=6.9 \mathrm{~Hz}, 2 \mathrm{H}), 7.59$ $7.66(\mathrm{~m}, 2 \mathrm{H}), 8.25(\mathrm{~s}, 1 \mathrm{H}), 13.05(\mathrm{~s}, 1 \mathrm{H})$. Anal. Calcd for $\mathrm{C}_{17} \mathrm{H}_{14} \mathrm{~N}_{2} \mathrm{O}_{3}: \mathrm{C}, 69.38 ; \mathrm{H}, 4.79 ; \mathrm{N}$, 9.52. Found: C, 69.62; H, 4.95; N, 9.31.

General procedure for preparation ethyl 1-alkyl-5-phenylindole-2-carboxylates (13a-c). To a suspension of $\mathrm{KOH}(0.28 \mathrm{~g}, 5 \mathrm{mmol})$ ethyl 5-phenylindole-2-carboxylate $6(0.4 \mathrm{~g}, 1.5 \mathrm{mmol})$ was added in DMSO $(2.5 \mathrm{ml})$ and the mixture stirred for $1 \mathrm{~h}$ at room temperature, then alkyl halide 12a-c ( $4 \mathrm{mmol}$ ) was added, the mixture stirred for $5 \mathrm{~h}$ and then diluted with water (300 $\mathrm{ml}$ ). The precipitate was filtered off, washed with water until $\mathrm{pH} 7$ and dried to give the corresponding 1-alkyl-2-ethoxycarbonyl-5-phenylindoles 13a-c (Table 2). 
Ethyl $\boldsymbol{N}$-methyl-5-phenylindole-2-carboxylate 13a. Yield 90\%; colorless crystals, mp 118-119 ${ }^{\circ} \mathrm{C} ; \mathrm{R}_{\mathrm{f}} 0.38$ benzene : hexane = 1:1 (v:v); UV $\lambda_{\max } / \mathrm{nm}$ (Ethanol), (loge) 207 (4.3), 262 (4.5), 302 (4.1). IR (KBr) $v_{\max } / \mathrm{cm}^{-1}: 1713(\mathrm{C}=\mathrm{O}), 2810\left(\mathrm{~N}_{-} \mathrm{CH}_{3}\right) .{ }^{1} \mathrm{H}$ NMR $\left(\mathrm{DMSO}-\mathrm{D}_{6}, 500 \mathrm{MHz}\right) \delta 1.34$ $(\mathrm{t}, J=7.8 \mathrm{~Hz}, 3 \mathrm{H}), 4.04(\mathrm{~s}, 3 \mathrm{H}), 4.32$ (q, $J=7.8 \mathrm{~Hz}, 2 \mathrm{H}), 7.32(\mathrm{~s}, 1 \mathrm{H}), 7.33(\mathrm{~d}, J=7.3 \mathrm{~Hz}, 1 \mathrm{H})$, 7.46-7.68 (m, 5H), $7.68(\mathrm{~d}, J=7.3 \mathrm{~Hz}, 1 \mathrm{H}), 7.95(\mathrm{~s}, 1 \mathrm{H}) ;{ }^{13} \mathrm{C}$ NMR (DMSO- $\left.d_{6}, 125 \mathrm{MHz}\right) \delta$ 14.2, 31.7, 60.4, 109.9, 111.4, 120.0, 124.3, 126.7, 128.8, 132.9, 138.8, 140.8, 161.2, 206.9. Anal. Calcd for $\mathrm{C}_{18} \mathrm{H}_{17} \mathrm{NO}_{2}$ : C, 77.40; H, 6.13; N, 5.01. Found: C, 77.64; H, 6.42; N, 5.33.

Ethyl $\boldsymbol{N}$-ethyl-5-phenylindole-2-carboxylate $13 \mathrm{~b}$. Yield $92 \%$; colorless crystals, mp 87-88 ${ }^{\circ} \mathrm{C}$; $\mathrm{R}_{\mathrm{f}} 0.39$ benzene: hexane $=1: 1,(\mathrm{v}: \mathrm{v}) ; \mathrm{UV} \lambda_{\max } / \mathrm{nm}$ (ethanol), (loge) 267 (4.28), 310 (3.83), 325 (3.64). IR (Vaseline oil) $v_{\max } / \mathrm{cm}^{-1}: 2933\left(\mathrm{~N}-\mathrm{C}_{2} \mathrm{H}_{5}\right), 1690(\mathrm{C}=\mathrm{O}) .{ }^{1} \mathrm{H}$ NMR (DMSO-D 6,300 $\mathrm{MHz}) \delta 1.40(\mathrm{t}, J=8.6 \mathrm{~Hz}, 3 \mathrm{H}), 1.44(\mathrm{t}, J=8.6 \mathrm{~Hz}, 3 \mathrm{H}), 4.36(\mathrm{q}, J=8.6 \mathrm{~Hz}, 2 \mathrm{H}), 4.64(\mathrm{q}, J=$ $8.6 \mathrm{~Hz}, 2 \mathrm{H}), 7.24(\mathrm{~s}, 1 \mathrm{H}), 7.26(\mathrm{t}, J=8.03,1 \mathrm{H}), 7.38(\mathrm{t}, J=8.03 \mathrm{~Hz}, 2 \mathrm{H}), 7.46(\mathrm{~d}, J=9.1 \mathrm{~Hz}$, $1 \mathrm{H}), 7.56(\mathrm{dd}, J=1.6 \mathrm{~Hz}, J=9.1 \mathrm{~Hz}, 1 \mathrm{H}), 7.59(\mathrm{dd}, J=2.6 \mathrm{~Hz}, J=8.03 \mathrm{~Hz}, 2 \mathrm{H}), 7.82(\mathrm{~d}, J=$ $1.6 \mathrm{~Hz}, 1 \mathrm{H}$ ); ${ }^{13} \mathrm{C}$ NMR (DMSO- $\left.d_{6}, 75 \mathrm{MHz}\right) \delta 13.9,15.2,59.6,109.9,110.1,119.9,124.1$, 125.9, 126.5, 128.1, 133.2, 137.5, 141.1. Anal. Calcd for $\mathrm{C}_{19} \mathrm{H}_{19} \mathrm{NO}_{2}$ : C, 77.79; H, 6.53; N, 4.74. Found: C, 77.787; H, 6.38; N, 4.92 .

Ethyl $\boldsymbol{N}$ - $\boldsymbol{n}$-Butyl-5-phenylindole-2-carboxylate 13c. Yield 87\%; colorless crystals, mp 112-113 ${ }^{\circ} \mathrm{C} ; \mathrm{R}_{\mathrm{f}} 0.40$ benzene: hexane $=1: 1,(\mathrm{v}: \mathrm{v}) ; \mathrm{UV} \lambda_{\max } / \mathrm{nm}$ (Ethanol), (loge) 263 (4.4), 259 (4.7), 301 (4.1). IR (Vaseline oil) $v_{\max } / \mathrm{cm}^{-1}: 2934\left(\mathrm{~N}-\mathrm{C}_{4} \mathrm{H}_{9}\right), 1695(\mathrm{C}=\mathrm{O}) .{ }^{1} \mathrm{H}$ NMR (DMSO- $\left.d_{6}, 300 \mathrm{MHz}\right)$ $\delta 1.0(\mathrm{t}, J=7.2 \mathrm{~Hz}, 3 \mathrm{H}), 1.35-1.43(\mathrm{~m}, 5 \mathrm{H}), 1.68(\mathrm{q}, J=7.3 \mathrm{~Hz}, 2 \mathrm{H}), 4.36(\mathrm{q}, J=7.2 \mathrm{~Hz}, 2 \mathrm{H})$, $4.59(\mathrm{t}, J=7.3 \mathrm{~Hz}, 2 \mathrm{H}), 7.24(\mathrm{~s}, 1 \mathrm{H}), 7.26(\mathrm{t}, J=7.1 \mathrm{~Hz}, 1 \mathrm{H}), 7.39(\mathrm{t}, J=7.1 \mathrm{~Hz}, 2 \mathrm{H}), 7.48(\mathrm{~d}, J$ $=8.7 \mathrm{~Hz}, 1 \mathrm{H}), 7.53(\mathrm{dd}, J=1.6 \mathrm{~Hz}, J=8.7 \mathrm{~Hz}, 1 \mathrm{H}), 7.59(\mathrm{dd}, J=1.2 \mathrm{~Hz}, J=7.9 \mathrm{~Hz}, 2 \mathrm{H}), 7.8$ $(\mathrm{d}, J=1.8 \mathrm{~Hz}, 1 \mathrm{H}) ;{ }^{13} \mathrm{C}$ NMR (DMSO-D, $\left.75 \mathrm{MHz}\right) \delta 15.4,20.5,29.4,54.6,109.7,111.5,115.6$, 116.5, 122.9, 125.2, 126.8, 132.2, 135.5, 140.2, 145.2. Anal. Calcd for $\mathrm{C}_{21} \mathrm{H}_{23} \mathrm{NO}_{2}$ : C, 78.50; $\mathrm{H}$, 7.17; N, 4.36. Found: C, 78.51; H, 7.18; N, 4.37.

8-phenyl-3,5-dihydro-4H-pyridazino[4,5-b]indol-4-one 15 . To the suspension of 2-ethoxycarbonyl-3-formyl-5-phenylindole $6(0.147 \mathrm{~g}, 5 \mathrm{mmol})$ in glacial acetic acid $(20 \mathrm{ml})$ hydrazine hydrate $(0.5 \mathrm{~g}, 10 \mathrm{mmol})$ was added and was stirred for $30 \mathrm{~min}$ at $\mathrm{rt}$. The formed yellow suspension was boiled for $1.5 \mathrm{~h}$. The precipitate was filtered off, washed with acetic acid, then with water until $\mathrm{pH} 7$, dried and recrystallized from dimethylformamide. Yield 92\%; colorless crystals (from dimethylformamide); $\mathrm{mp} 275^{\circ} \mathrm{C}$ decomp.; $\mathrm{R}_{\mathrm{f}} 0.29$ ethanol; $\mathrm{UV} \lambda_{\max } / \mathrm{nm}$ (ethanol), (loge) 275 (3.92), 320 (3.25), 330 (3.19); IR (vaseline oil) $v_{\max } / \mathrm{cm}^{-1}: 3440-3500(\mathrm{NH}), 1670$ $(\mathrm{C}=\mathrm{O}), 1620(\mathrm{C}=\mathrm{N}) ;{ }^{1} \mathrm{H} \mathrm{NMR}\left(\mathrm{DMSO}_{6}, 500 \mathrm{MHz}\right) \delta 7.35(\mathrm{t}, J=7.6,1 \mathrm{H}) ; 7.49(\mathrm{t}, J=7.6$, $2 \mathrm{H}) ; 7.69(\mathrm{~d}, J=8.5,1 \mathrm{H}) ; 7.76(\mathrm{~d}, J=7.6,2 \mathrm{H}) ; 7.83(\mathrm{~d}, J=8.5,1 \mathrm{H}) ; 8.51(\mathrm{~s}, 1 \mathrm{H}) ; 8.83(\mathrm{~s}, 1 \mathrm{H})$; $12.78(\mathrm{~s}, 1 \mathrm{H}) ; 12.84(\mathrm{~s}, 1 \mathrm{H}) ;{ }^{13} \mathrm{C}$ NMR (DMSO- $\left.d_{6}, 125 \mathrm{MHz}\right) \delta 187.59,160.29,140.91,136.26$, 135.47, 133.46, 129.17, 128.96, 127.09, 126.68, 120.26, 120.06, 118.78, 113.88, 113.66, 61.59, 14.08. Anal. Calcd for $\mathrm{C}_{16} \mathrm{H}_{11} \mathrm{~N}_{3} \mathrm{O}$ : C, 73.56; H, 4.21; N, 16.09. Found: C, 73.65; H, 4.31; N, 16.04 . 


\section{Acknowledgements}

This project has been supported by finance from the Georgia National Science Foundation (Grant №GNSF/ST07/4-181) Any ideas in this publication represent those of the author and may not represent the opinion of the Georgia National Science Foundation itself. We also want to thank the Deutsche Akademische Austauschdienst (DAAD) for supporting the partnership and the exchange program between the Ivane Javakhishvili University in Tbilisi and the Saarland University.

\section{References}

1. Barton, D.; Ollis, W. D. Comprehensive Organic Chemistry; Khimia: Moscow, 1985, Vol. 8, p 751 .

2. (a) Gerasimenko, I.; Sheludko, Y.; Stockigt, J. J. Nat. Prod. 2001, 64, 114. (b) Katritzky, A. R.; Rees, C. W.; Scriven, E. F. V. Comprehensive Heterocyclic Chemistry, 1996, 2, 207. (c) Sarges, R.; Howard, H. R.; Koe, B. K.; Weissman, A. J. Med. Chem. 1989, 32, 437.

3. (a) Houlihan, W. J. U.S. Patent. 3987 059, 1977; Chem. Abstr. 1977, 86, 7, 43558f. (b) Lounasmaa, M., Nemest, A. Tetrahedron 1982, 38, 2, 223. (c) Srivastava, N.; Sengupta, A. K. Indian J. Chem. Sec. B. 1983, 7, 707.

4. (a) Samsoniya, Sh. A.; Targamadze, N. L.; Suvorov, N. N. Russ. Chem. Rev., 1994, 63, 10, 866. (b) Chikvaidze, I. Sh.; Mumladze E. A.; Samsoniya, Sh. A.; Suvorov, N. N. J. Pharm. Chem. 1994, 28, 47.

5. (a) Inion, H.; De Vogelaer, H.; Decamps, M., Bauthier, J.; Colot, M.; Richard, J. Eur. J. Med. Chem. Chim. Ther. 1977, 12, 483. (b) Inion, H.; De Vogelaer, H.; Van Durme, E., Decamps, M.; Brotelle, R.; Charlier, R.; Colot, M. Eur. J. Med. Chem. Chim. Ther. 1975, 10, 276.

6. (a) Yama-kawa, Y.; Koggo, K. K. Japan Patent. 80122 703, 1980; Chem. Abstr. 1980, 93, 25, 232727. (b) Drugs of the Future 1985, 10, 1029. (c) Tamblyn, J. W. U.S. Patent. 3240 752, 1966; Chem. Abstr. 1966, 64, 15, 16086b.

7. Pigerol, Ch.; De Cointel de Fillain, P.; Eymard, P.; Werbence, J. P.; Boll, M. Ger. Offen. 2 727 047, 1978; Chem. Abstr. 1978, 88, 15, 105132q.

8. (a) Arheidt K. Liebig's Ann. Chem. 1887, 239, 206. (b) Chikvaidze, I. Sh.; Samsoniya, Sh. A.; Kozik T. A.; Suvorov, N. N. Chem. Heterocycl. Compd. 1980, 1508. (c) Samsoniya, Sh. A.; Tabidze D. M.; Suvorov, N. N. Chem. Heterocycl. Compd. 1981, 57. (d) Samsoniya, Sh. A.; Tabidze D. M.; Suvorov, N. N. J. Org. Chem. 1981, 51, 1436.

9. (a) Mirziashvili N. T.; Chikvaidze, I. Sh.; Kapanadze T. G.; Samsoniya, Sh. A.; Suvorov, N. N. Chem. Heterocycl. Compd. 1991, 54. (b) Chikvaidze, I. Sh.; Megrelishvili N. Sh.; Samsoniya, Sh. A.; Suvorov, N. N. Chem. Heterocycl. Compd 1991, 1508. (c) Mumladze E. A.; Chikvaidze, I. Sh.; Samsoniya, Sh. A.; Kozik T. A.; Suvorov, N. N. Proc.Acad. Sci. Georgia 1985, 119, 2, 313. (d) Gogrichiani E. O.; Chikvaidze, I. Sh.; Jibladze L. I.; Cotodze 
M. V.; Samsoniya, Sh. A.; Suvorov, N. N. Chem. Heterocycl. Compd 1994, 1351. (e) Chikvaidze, I. Sh.; Gogrichiani E. O.; Kupkovskaia L. N.; Baramidze L. V.; Samsoniya, Sh. A. Chem. Heterocycl. Compd 1993, 1055. (f) Chikvaidze, I. Sh.; Narimanidze N. O.; Samsoniya, Sh. A.; Suvorov, N. N. Chem. Heterocycl. Compd 1993, 1194. (g) Chikvaidze, I. Sh.; Samsoniya, Sh. A.; Narindoshvili T. G. Chem. Heterocycl. Compd. 2004, 524.

10. Chikvaidze, I. Sh.; Megrelishvili N. Sh.; Samsoniya, Sh. A.; Suvorov, N. N. J. Pharm. Chem. 1998, 32, 1, 30 .

11. Samsoniya, Sh. A.; Chikvaidze, I. Sh.; Narindoshvili T. G. Selected methods for synthesis and modifikation of heterocycles, Kartsev, V. G. Ed.; IBS Press: Moscow 2004, Vol. 3, pp 219-259.

12. (a) Golob, T.; Liebi, R.; Angerer, E. Bioorg. Chem. Med. Chem. 2002, 10, 3941. (b) Gao H.; Katzenellenbogen J. A.; Garg R. Chem. Rev. 1999, 99, 723. (c) Chalut K. J.; Ostrander J. H.; Giacomelli M. G. Wax A. Cancer Research 2009, 69, 1199. (d) Azmi S.; Wang Zh.; Burikhanov R.; Rangnekar V. M.; Wang G.; Chen J.; Sarkar F. H.; Mohammad R. M. Mol. Cancer Ther. 2008, 7, 9, 2884. (e) Yoon J. S.; Seo J. Ch.; Han S. W. Am. J. Chinese Medicine 2006, 34, 3, 401.

13. Agarwal A.; Pearson P. P.; Taylor E. W.; Li H. B.; Dahlgren T.; Heralof M.; Yang Y.; Lambert G.; Nelson D. L.; Regan J. W.; Martin A. R. J. Med. Chem. 1993, 36, 4006.

14. Elokdan H.; Abou-Gharbia M.; Hennan J. K.; Mc Farlane G.; Mugford C. P.; Krishnamurthy G.; Crandall D. L. J. Med. Chem. 2004, 47, 3491.

15. (a) Kitaev, Iu. P.; Troepolskaia T. V. Chemistry of Heterocyclic Compounds 1978, 8, 1011.

(b) Wagaw, S.; Yang, B. H.; Buchwald, S. L. J. Am. Chem. Soc. 1999, 121, 10251. (c) Robinson, B. The Fischer Indole Synthesis; Willey: New York, 1989, p 923.

16. Grible, G. W. J. Chem. Soc., Perkin Trans. 1 2000, 1045.

17. Suvorov, N. N.; Samsoniya, Sh. A.; Chilikin, L. G.; Chikvaidze, I. Sh.; Turchin, K. F.; Efimova, T. K.; Tretiakova, L. G.; Gverdciteli, M. I. Chem. Heterocycl. Compd. 1978, 2, 217.

18. Jungietu, G. N.; Budilin, V. A.; Kost, A. N. Preparation Chemistry of Indole; Shtiintsa: Kishiniov, 1975; p 264.

19. Tramontini, M. Synthesis 1973, 12, 703.

20. (a) Beliaev, E. IU.; Gudaspov, B. V. Aromatic Nitrosocompounds; Leningrad: Khimia, 1989, p 172. (b) Jackson, A. H.; Johnston, D. N.; Shannon, P. N. P. J. Chem. Soc., Perkin Trans. 1 1977, 1024. (c) Shagalov, L. B.; Tkachenko, T. A.; Vasilev, A.M.; Eraksina, V. N.; Babushkina, T. A.; Suvorov, N.N. Chem. Heterocycl. Compd. 1974, 7, 940 (d) Smith, J.; Perrin, C.; Langlois, M.; Suquet, M. Bull. Soc. Chim. Fr., 1969, 4, 1227. (e) Barton, D.; Ollis, W.D. Comprehensive Organic Chemistry; Khimia: Moscow, 1985, Vol. 8, pp 500-502. (f) Samsoniya, Sh. A., Maharramov, M. A., Targamadze, N. L., Samsonia, N. Sh., Mumladze, E. A., Chikvaidze, I. Sh. Proc. Georgian Nat. Acad. Sci. 2008, 34, 40.

21. (a) Avramenko, V. G.; Nazina, V. D.; Suvorov, N. N. Chemistry of Heterocyclic Compounds 1970, 8, 1071. (b) Srivastava, N., Sengupta, A. K. Indian J. Chem. 1983, 22B, 707. 
22. (a) Heaney, H., Ley, S. V. J. Chem. Soc., Perkin Trans. 1 1973, 499. (b) Obinochni, A. A.; Anisimov, B. N.; Sergeeva, N. D.; Prostakov, N. S. Chem. Heterocycl. Compd. 1993, 5, 648. 23. Allen, C. F. H., Young, D. M., Gilbert, M. R. J. Org. Chem. 1937, 2, 235. 